\title{
GRB 070610: A CURIOUS GALACTIC TRANSIENT
}

\author{
M. M. Kaslinal, ${ }^{1}$ S. B. Cenko, ${ }^{2}$ S. R. Kulkarni, ${ }^{1}$ P. B. Cameron, ${ }^{1}$ E. Nakar, ${ }^{1}$ E. O. Ofek, ${ }^{1}$ A. Rau, ${ }^{1}$ \\ A. M. Soderberg, ${ }^{1}$ S. Campana, ${ }^{3}$ J. S. Bloom, ${ }^{4}$ D. A. Perley,${ }^{4}$ L. K. Pollack,${ }^{5}$ S. Barthelmy ${ }^{6}$ \\ J. Cummings, ${ }^{6}$ N. Gehrels, ${ }^{6}$ H. A. Krimm, ${ }^{7,8}$ C. B. Markwardt, ${ }^{7,9}$ G. Sato, ${ }^{6}$ P. Chandra, ${ }^{10}$ \\ D. Frail ${ }^{11}$ D. B. Fox, ${ }^{12}$ P. A. Price, ${ }^{13}$ E. Berger, ${ }^{14,15}$ S. A. Grebenev, ${ }^{16}$ \\ R. A. Krivonos, ${ }^{16,17}$ and R. A. Sunyaev ${ }^{15,16}$ \\ Received 2007 August 1; accepted 2007 November 5
}

\begin{abstract}
GRB 070610 is a typical high-energy event with a duration of $5 \mathrm{~s}$. Yet within the burst localization we detect a highly unusual X-ray and optical transient, Swift J195509.6+261406. We see high-amplitude X-ray and optical variability on very short timescales even at late times. Using near-infrared imaging assisted by a laser guide star and adaptive optics, we identified the counterpart of Swift J195509.6+261406. Late-time optical and near-infrared imaging constrain the spectral type of the counterpart to be fainter than a K-dwarf, assuming it is of Galactic origin. It is possible that GRB 070610 and Swift J195509.6+261406 are unrelated sources. However, the absence of a typical X-ray afterglow from GRB 070610 in conjunction with the spatial and temporal coincidence of the two motivate us to suggest that the sources are related. The closest (imperfect) analog to Swift J195509.6+261406 is V4641 Sgr, an unusual black hole binary. We suggest that Swift J195509.6+261406 along with V4641 Sgr define a subclass of stellar black hole binaries - the fast $\mathrm{X}$-ray novae. We further suggest that fast X-ray novae are associated with bursts of gamma rays. If so, GRB 070610 defines a new class of celestial gamma-ray bursts and these bursts dominate the long-duration GRB demographics.
\end{abstract}

Subject headings: gamma rays: bursts - stars: flare $-\mathrm{X}$-rays: binaries $-\mathrm{X}$-rays: bursts -

X-rays: individual (Swift J195509.6+261406)

Online material: machine-readable table

\section{DISCOVERY OF GRB 070610}

Launched in November 2004, the Swift Gamma-Ray Burst Explorer (Gehrels et al. 2004) was designed to localize gammaray bursts (GRBs) and undertake rapid and sustained X-ray and ultraviolet observations of the resulting afterglow. With over 200 events now localized and studied, Swift has made fundamental contributions to both long-duration soft bursts (LSBs) and shortduration hard bursts (SHBs). LSBs appear to trace cosmological massive-star formation rate with one event at a redshift of 6.3.

\footnotetext{
1 Division of Physics, Mathematics and Astronomy, California Institute of Technology, MS 105-24, Pasadena, CA 91125.

2 Space Radiation Laboratory, California Institute of Technology, MS 220-47, Pasadena, CA 91125.

3 INAF, Osservatorio Astronomica di Brera, via E. Bianchi 46, I-23807 Merate (LC), Italy.

${ }_{5}^{4}$ Department of Astronomy, University of California, Berkeley, CA 94720.

5 Department of Astronomy and Astrophysics, University of California, Santa Cruz, CA 95064.

6 NASA Goddard Space Flight Center, Greenbelt, MD 20771.

7 CRESST and Astroparticle Physics Laboratory, NASA/GSFC, Greenbelt, MD 20771.

${ }^{8}$ Universities Space Research Association, 10211 Wincopin Circle, Suite 500, Columbia, MD 21044.

9 Department of Astronomy, University of Maryland, College Park, MD 20742.

${ }^{10}$ University of Virginia, P.O. Box 400325, Charlottesville, VA 22903.

11 National Radio Astronomy Observatory, Socorro, NM 87801.

12 Department of Astronomy and Astrophysics, 525 Davey Laboratory, Pennsylvania State University, University Park, PA 16802.

13 Institute for Astronomy, University of Hawaii, 2680 Woodlawn Drive, Honolulu, HI 96822.

14 Observatories of the Carnegie Institute of Washington, Pasadena, CA 91101.

15 Princeton University Observatory, Princeton, NJ 08544.

16 Space Research Institute, Profsoyuznaya 84/32, 117997 Moscow, Russia.

17 Max-Plank-Institut für Astrophysik, Karl-Schwarzschild-Strasse 1, D-85741 Garching, Germany.
}

SHBs have been seen at typical redshifts of $\sim 0.5$ in both elliptical and star-forming galaxies. There is now some circumstantial evidence for SHBs being the result of coalescence of compact objects.

At $20: 52: 26$ UT on 2007 June 10 the Burst Alert Telescope (BAT; Barthelmy et al. 2005) aboard Swift triggered on GRB 070610. The high-energy prompt emission had a duration $\left(T_{90}\right)$ of $4.6 \mathrm{~s}$ (Pagani et al. 2007b). Over the range $15-150 \mathrm{keV}$ the burst could be fitted with a power law with photon index $\Gamma=1.76 \pm$ 0.25 , resulting in a fluence of $(2.4 \pm 0.4) \times 10^{-7} \mathrm{ergs} \mathrm{cm}{ }^{-2}$ (Tueller et al. 2007). A blackbody model is inconsistent with this emission (reduced $\chi^{2}=1.7$ ).

The burst profile consisted of a single symmetric peak (Fig. 1). Fitting the profile (Norris et al. 1996), we calculate a rise time (i.e., half-width at half-maximum) of $1.68 \pm 0.55 \mathrm{~s}$. As can be seen from Figure 2, the duration and the hardness ratio of Swift J195509.6+261406 are both consistent with the broader population of extragalactic long-duration GRBs observed by Swift.

The BAT localized GRB 070610 to $\alpha=19^{\mathrm{h}} 55^{\mathrm{m}} 13.1^{\mathrm{m}}, \delta=$ $+26^{\circ} 15^{\prime} 20^{\prime \prime}(\mathrm{J} 2000.0)$ and a $90 \%$ containment radius of $1.8^{\prime}$. As can be seen in Figure 3 the field is dense, which is not surprising given the Galactic location $\left(l=63.3^{\circ}\right.$ and $\left.b=-1.0^{\circ}\right)$.

Here we report the discovery of an unusual X-ray transient (hereafter referred to as Swift J195509.6+261406) in the error circle of GRB 070610 and follow-up optical, near-infrared (NIR) and radio observations.

\section{Swift J195509.6+261406: A TRANSIENT X-RAY SOURCE}

The X-Ray Telescope (XRT; Burrows et al. 2005) began observing the field of GRB $0706103.2 \mathrm{ks}$ after the initial BAT trigger (prompt slewing was disabled due to an Earth limb constraint). The XRT detected a single uncataloged variable source in the BAT error 


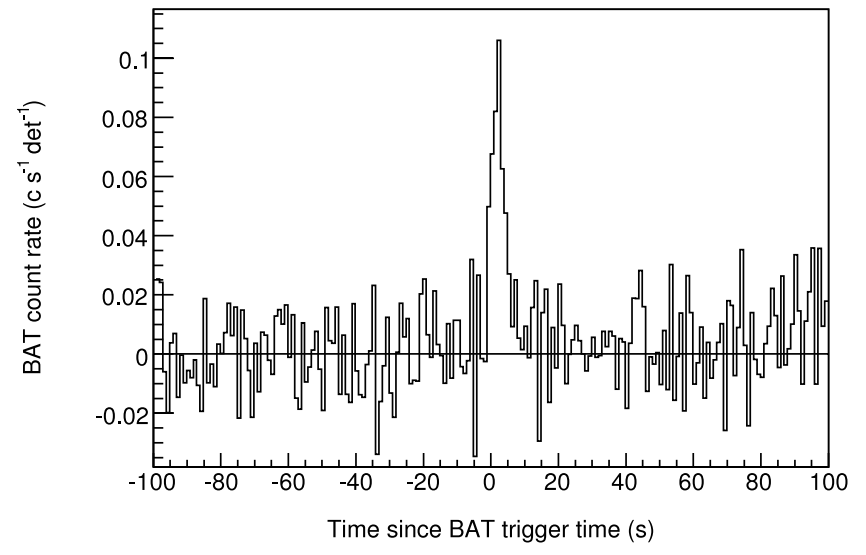

FIG. 1.- Swift BAT light curve of GRB 070610, at 15-150 keV, with $1 \mathrm{~s}$ time resolution. The conversion factor to translate the ordinate to cgs flux units is $5.6 \times$ $10^{-7}$ ergs $\mathrm{cm}^{-2} c^{-1} \operatorname{det}\left(1 \operatorname{det}=0.16 \mathrm{~cm}^{2}\right)$.

circle at $\alpha=19^{\mathrm{h}} 55^{\mathrm{m}} 9.6^{\mathrm{s}}, \delta=+26^{\circ} 14^{\prime} 6.7^{\prime \prime}(90 \%$ confidence error circle of $4.3^{\prime \prime}$ radius; Pagani et al. 2007a). This position was further refined to $\alpha=19^{\mathrm{h}} 55^{\mathrm{m}} 9.66^{\mathrm{s}}, \delta=+26^{\circ} 14^{\prime} 5.2^{\prime \prime}$ (90\% confidence error circle of $1.2^{\prime \prime}$ radius ${ }^{18}$ ).

The XRT continued to monitor Swift J195509.6+261406 over the course of the next month until the source was no longer detected.

The XRT data were processed with xrtpipeline (ver. 0.10.6). All data were obtained in photon counting mode. In this mode the entire CCD is read and the time resolution is limited to $2.5 \mathrm{~s}$. We extracted grade 0-12 events (Burrows et al. 2005) from a 15 pixel radius circular region centered on the source. To account for the background, we extracted events within a 40 pixel radius circular region in the vicinity of the transient but not encompassing any other source in the field. We adaptively extracted the light curve binning the data in order to have 10 counts per bin. The light curve was corrected for the extraction region losses and for CCD defects as well as for vignetting by using the task xrtlccorr (ver. 0.1.9), which generates an orbit-by-orbit correction based on the instrument map.

The X-ray light curve of Swift J195509.6+261406 is shown in Figure 4 and compared to a small sample of long-duration GRB afterglows in Figure 5. Kann et al. 2007 were the first to suggest that this GRB was likely to be of Galactic origin. Clearly, Swift J195509.6+261406 differs from typical GRB X-ray afterglows in two fundamental respects. First, it does not exhibit the strong (overall) secular decrease in flux over timescales of hours (Nousek et al. 2006; Zhang et al. 2006). While the decay index in longduration GRBs can vary markedly from one phase to another, Swift J195509.6+261406 shows no significant decline until very late times $\left(\sim 10^{6} \mathrm{~s}\right)$.

Second, the XRT light curve of Swift J195509.6+261406 consists of spikes - never seen before in any afterglow. In particular we draw the attention of the reader to a dramatic flare at $t \sim$ $7.86 \times 10^{4} \mathrm{~s}$, jumping by a factor of $\Delta f / f \sim 100$ in flux over a timescale of $\Delta t / t \sim 10^{-4}$ (see Fig. 4 , inset). None of the 69 XRT flares described in Chincarini et al. (2007) exhibit a comparable amplitude spike at late time. While a strong X-ray flare has been seen in GRB 050502B (Falcone et al. 2006) (see Fig. 5) the fractional duration, $\Delta t / t$ is much larger $(\sim 0.5)$. Less significant variability is present throughout the duration of observations of Swift J195509.6+261406.

${ }^{18}$ See http://astro.berkeley.edu/ nat/swift/xrt_pos.html.

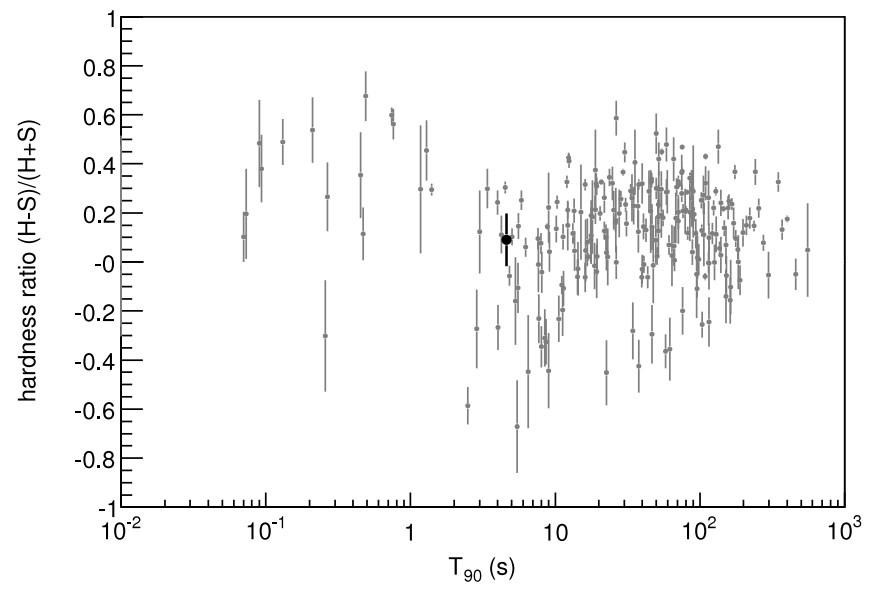

FIG. 2.-Plot of duration $\left(T_{90}\right)$ and hardness ratio (HR) of 226 Swift bursts from GRB 041217 to GRB 070616. We define hardness ratio as $(H-S) /(H+S)$, where $S$ and $H$ are energy fluences in $15-50$ and $50-150 \mathrm{keV}$, respectively. The values of $T_{90}$ and hardness ratio for GRB 070610 (large filled black circle) are $4.6 \pm 0.4 \mathrm{~s}$ and $0.09 \pm 0.11(90 \%$ confidence level $)$, respectively.

We searched the XRT flare for pulsations, and 521 photons were extracted within $60^{\prime \prime}$ of the source position and corrected to the solar system barycenter with the task barycorr. To search for pulsations we constructed the $Z_{1}^{2}$ power spectrum to a maximum frequency of $0.2 \mathrm{~Hz}$ (Buccheri et al. 1983). The largest observed value of $Z_{1}^{2}$ was 25.2 at a frequency of $0.1446 \mathrm{~Hz}$. Since $Z_{n}^{2}$ is distributed as $\chi^{2}$ with $2 n$ degrees of freedom, this value corresponds to a single trial detection significance of $4.8 \sigma$ in equivalent Gaussian units. Given that we have performed 350 trials, the significance of this detection is $3.4 \sigma$, and thus we do not consider this result to be conclusive evidence of periodicity.

For spectral analysis the ancillary response files were generated with the task xrtmkarf. We used the latest spectral redistribution matrices (ver. 009). Data were extracted from single or consecutive orbits in order to have at least 100 counts per spectrum. Spectra were binned to a minimum of 15 counts per energy bin. The resulting spectra were inconsistent with a blackbody (reduced $\chi^{2}=$ 1.9) and consistent with a power-law model (task phabs). The best-fit column density $\left(N_{\mathrm{H}}\right)$ and photon index $(\Gamma)$ for each epoch are summarized in Table 1 . Overall, we find that the inferred flux conversion is approximately 1 count s${ }^{-1} \approx 1.3 \times 10^{-10} \mathrm{ergs} \mathrm{cm}^{-2}$ $\mathrm{s}^{-1}$ in the $0.3-10 \mathrm{keV}$ band.

We extrapolate the XRT flare spectrum to BAT $(15-50 \mathrm{keV})$ and predict a flux of $1.8 \times 10^{-9} \mathrm{ergs} \mathrm{cm}^{-2} \mathrm{~s}^{-1}$. This corresponds to a BAT count rate of 0.0032 counts $\mathrm{s}^{-1} \mathrm{det}^{-1}$. This is consistent with a $2 \sigma$ upper limit from two $64 \mathrm{~s}$ intervals of BAT data straddling the XRT flare -0.0038 counts s${ }^{-1} \operatorname{det}^{-1}$ (at $78499.8 \mathrm{~s}$ ) and 0.012 counts $\mathrm{s}^{-1} \mathrm{det}^{-1}$ (at $78563.8 \mathrm{~s}$ ).

The inferred interstellar extinction along this low Galactic latitude is quite high and thus uncertain: $N_{\mathrm{H}}$ of $1.1 \times 10^{22} \mathrm{~cm}^{-2}$ (Dickey \& Lockman 1990); $0.8 \times 10^{22} \mathrm{~cm}^{-2}$ (Kalberla et al. 2005); and (1.56-1.89) $\times 10^{22} \mathrm{~cm}^{-2}$ (Schlegel et al. 1998). The former two estimates are based on $\mathrm{H}$ I data, whereas the latter on diffuse infrared emission. Given the uncertainty in the inferred $N_{\mathrm{H}}$ the XRT spectrum cannot be used to determine the distance to Swift J195509.6+261406.

\section{A FLICKERING OPTICAL VARIABLE}

Rapid observations in response to the BAT trigger, in particular by the OPTIMA-Burst team (Stefanescu et al. 2007a), revealed a rapidly variable (timescales as low as tens of seconds) optical transient inside the XRT error circle of Swift J195509.6+261406. 


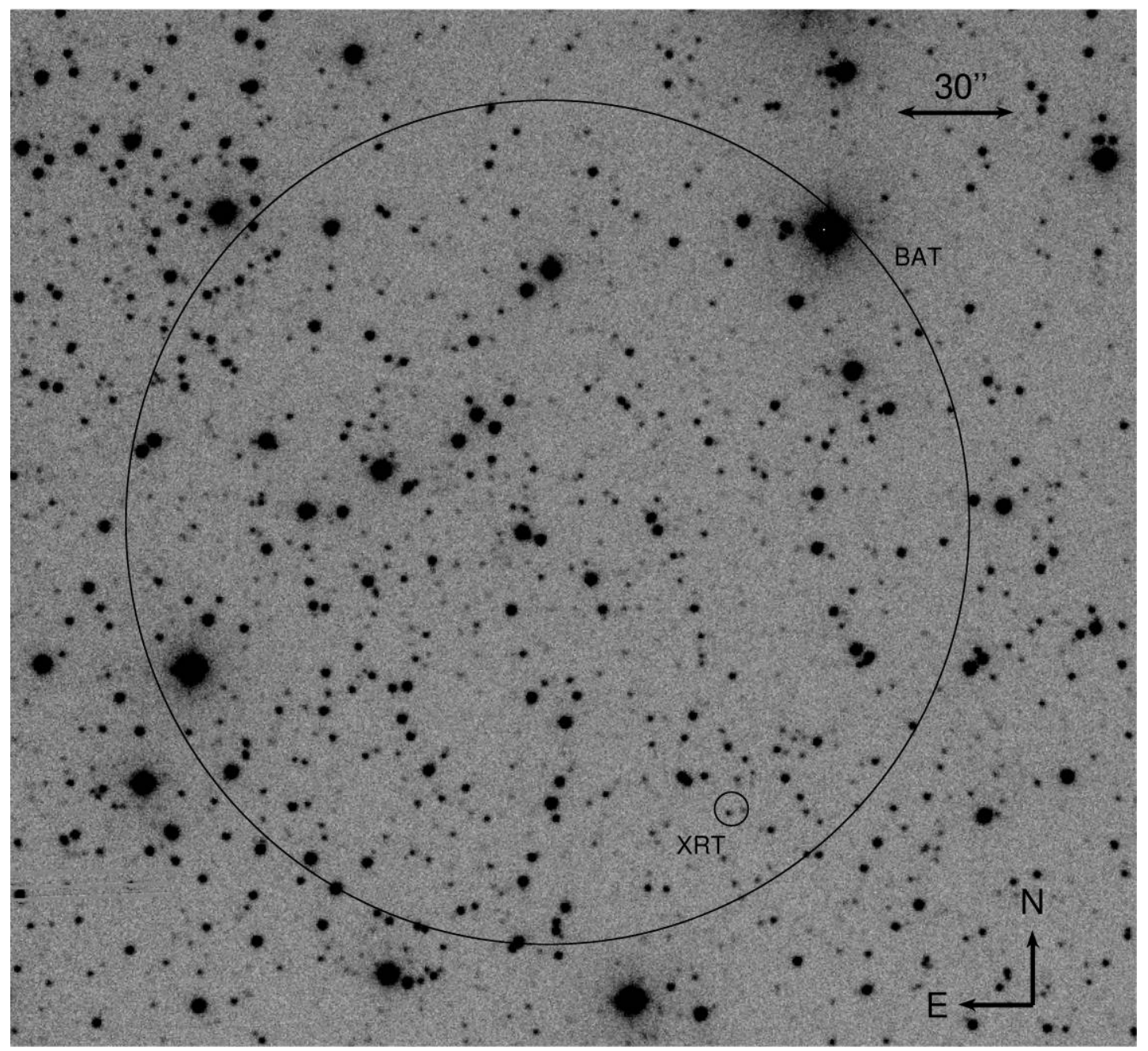

FIG. 3.- Optical image ( $i^{\prime}$-band) of the field of GRB 070610 obtained by the automated Palomar 60 inch telescope on UT 2007 June 12 . The BAT localization of GRB 070610 has a radius of $1.8^{\prime}$, while the XRT localization of Swift J195509.6+261406 has a radius of $4.3^{\prime \prime}$; both are indicated with black circles. The bright source in the XRT circle is Swift J195509.6+261406.

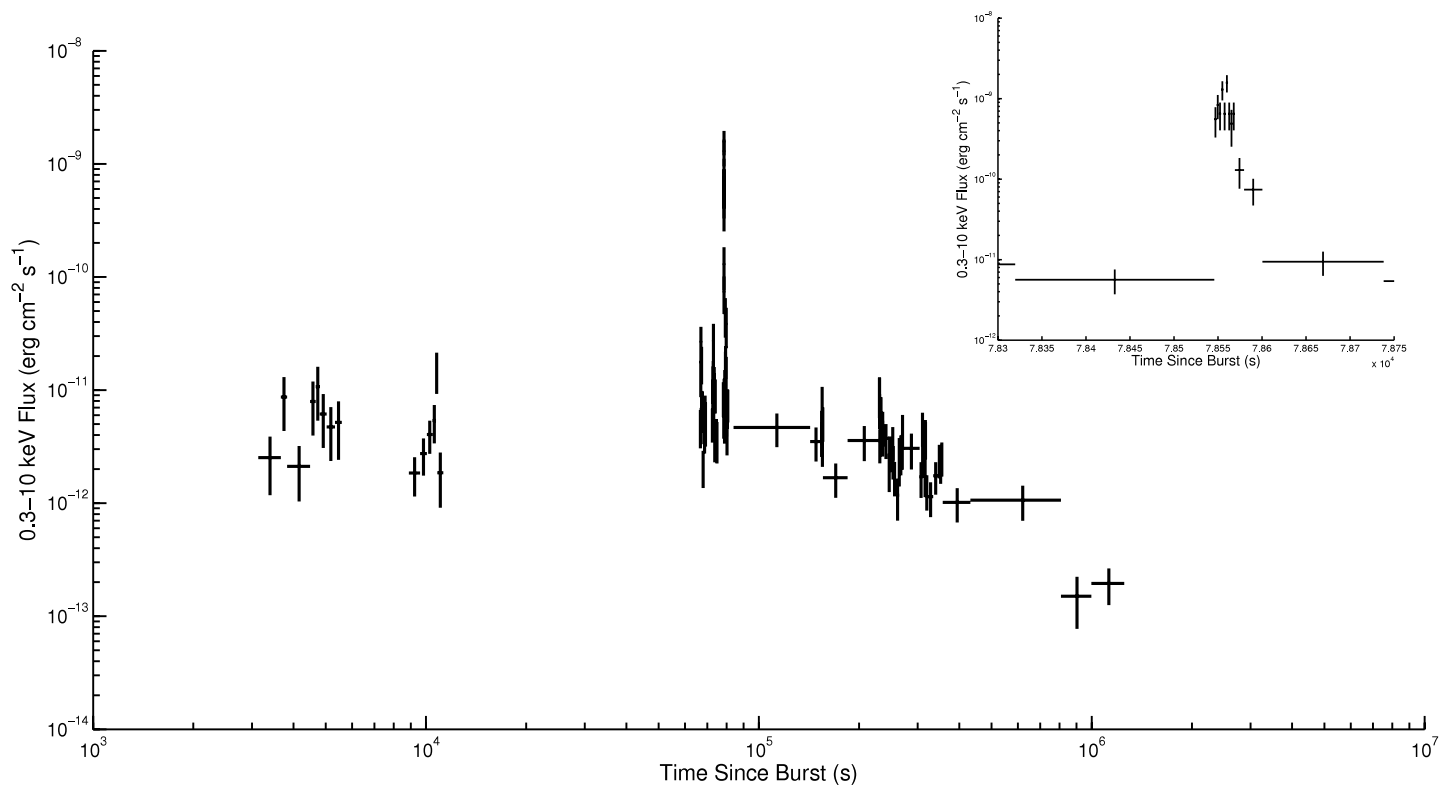

FIG. 4. - XRT light curve of Swift J195509.6+261406 in the energy band $0.3-10 \mathrm{keV}$. The dramatic X-ray flare at $t \sim 7.86 \times 10^{4} \mathrm{~s}$ is shown in the inset. 


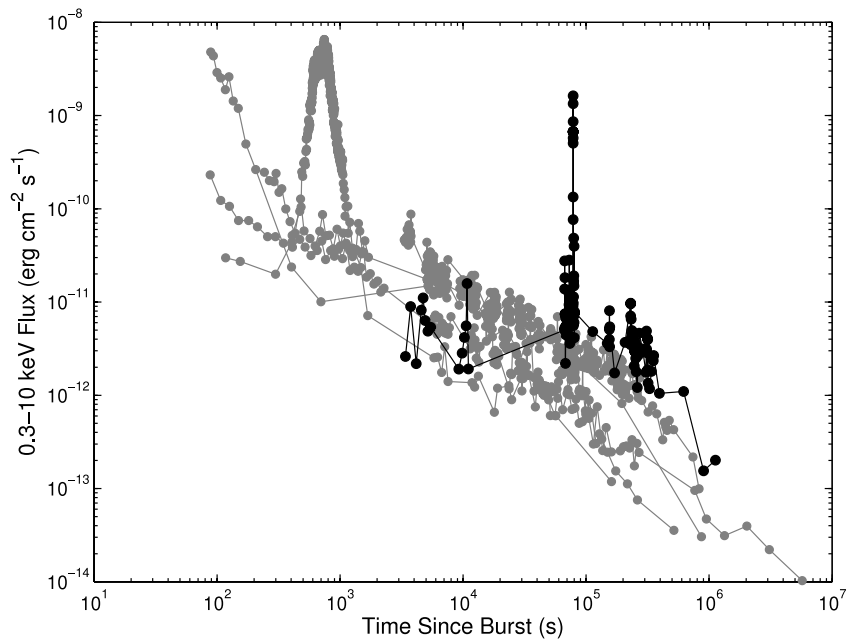

FIG. 5.-XRT light curves of a small sample of extragalactic long-duration GRBs (GRBs 050315, 050318, 050319, 050416A, and 050502B) are shown in gray. Data are from Evans et al. 2007. All show the approximately power-law decay typical of GRB afterglows. GRB 050502B exhibits a bright flare around $t \sim 10^{3} \mathrm{~s}$ (see Falcone et al. 2006). However, the rise time of this flare is much longer than the spike seen in Swift J195509.6+261406 (shown in black).

Astronomers using other facilities - including the OSN $1.5 \mathrm{~m}$ telescope (Postigo et al. 2007), the $2 \mathrm{~m}$ Schmidt Telescope of the Thüringer Landessternwarte (Kann et al. 2007), the $25-\mathrm{cm}$ TAROT facility (Klotz et al. 2007), and the $40 \mathrm{~cm}$ Watcher Telescope (French et al. 2007) - confirmed the detection of this variable source. Detections and upper limits reported to the GRB Coordinates Network $\left(\mathrm{GCN}^{19}\right)$ are shown in Figure 6.

Drawn by the excitement of these discoveries, we began monitoring the field of Swift J195509.6+261406 in the $i^{\prime}$ filter with the automated Palomar 60 inch telescope (P60; Cenko et al. 2006) starting at $5: 47$ UT 2007 June 12 and continued over the next several nights. In addition, we imaged the field in $R$-, $I$ - and $g$ - bands with the Low Resolution Imaging Spectrograph (LRIS; Oke et al. 1995) mounted at the Cassegrain focus of the Keck I 10 m telescope. All images were reduced using standard IRAF ${ }^{20}$ routines.

The light curve obtained from our observations is also summarized in Figure 6. The P60 and the Keck photometry can be found in Tables 2 and Table 3, respectively.

The P60 light curve is dominated by flickering and magnificent flares on the night of UT 2007 June 12 (see Fig. 7). We ob-

19 See http://gcn.gsfc.nasa.gov/gcn3_archive.html.

20 IRAF is distributed by the National Optical Astronomy Observatory, which is operated by the Association for Research in Astronomy, Inc., under cooperative agreement with the National Science Foundation.

TABLE 1

XRT Spectral Analysis

\begin{tabular}{|c|c|c|c|}
\hline $\begin{array}{l}\text { Epoch Start } \\
\text { (MJD) }\end{array}$ & $\begin{array}{c}\text { Total Exposure } \\
\text { (s) }\end{array}$ & $\begin{array}{c}N_{\mathrm{H}} \\
\left(10^{22} \mathrm{~cm}^{-2}\right)\end{array}$ & $\Gamma$ \\
\hline 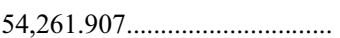 & 4811 & $0.30_{-0.23}^{+0.29}$ & $1.43 \pm 0.37$ \\
\hline 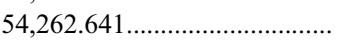 & 7912 & $0.76_{-0.18}^{+0.24}$ & $1.93 \pm 0.18$ \\
\hline $54,263.268,54,264.004 \ldots \ldots \ldots$ & 2947,10500 & $0.59_{-0.23}^{+0.18}$ & $1.11 \pm 0.22$ \\
\hline 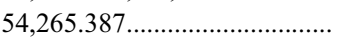 & 6026 & $0.61_{-0.33}^{+0.53}$ & $1.33 \pm 0.40$ \\
\hline 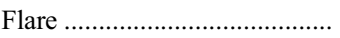 & $\ldots$ & $0.92_{-0.57}^{+0.91}$ & $1.74 \pm 0.48$ \\
\hline All but flare........ & $\ldots$ & $0.72_{-0.12}^{+0.14}$ & $1.71 \pm 0.11$ \\
\hline
\end{tabular}

NotE.-We have fit the XRT data to a power-law model of the form $N(E) \propto$ $E^{-\Gamma}$, leaving the line-of-sight $N_{\mathrm{H}}$ as a free parameter.

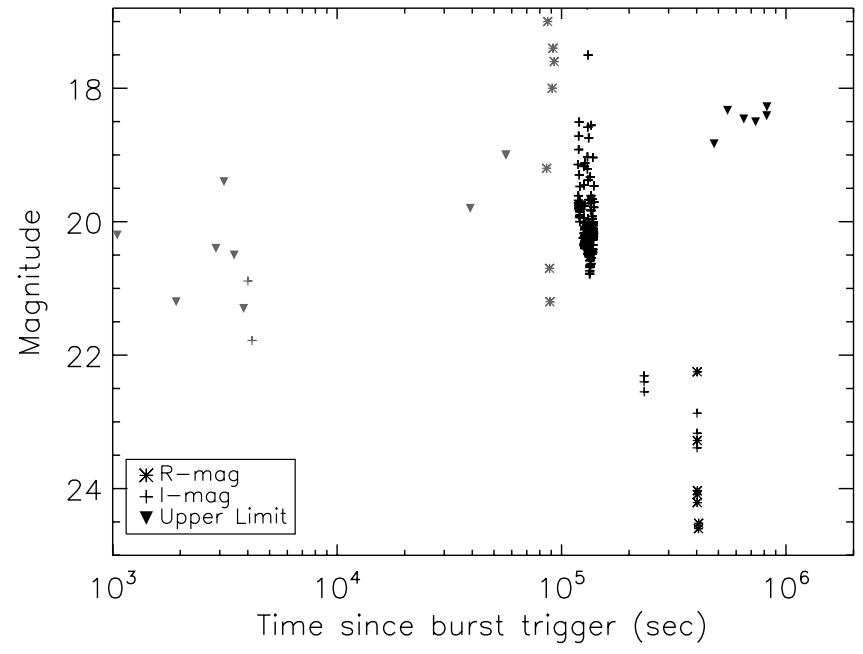

FIG. 6.-Optical light curve of Swift J195509.6+261406, including data from P60 (black), Keck/LRIS (black), and the literature (gray) (French et al. 2007; Postigo et al. 2007; Kann et al. 2007; Updike et al. 2007a, 2007b; Stefanescu et al. 2007b. 2007c; Yoshida et al. 2007; Klotz et al. 2007).

served over 11 flares with amplitudes greater than 1 mag in only $3 \mathrm{hr}$. The brightest of these flares rose and dropped by more than 3.5 mag within 6 minutes. The amplitude of the flares is a lower limit because the P60 images are not deep enough to detect the quiescent counterpart (see below). The timescale is also an upper limit because it is entirely possible that variability is more rapid than our sampling rate $(\sim 60 \mathrm{~s})$. If we define duty-cycle as the fraction of time for which the Swift J195509.6+261406 was brighter than $i^{\prime}<20$, then the duty cycle based on the first night of data is $18.6 \%$. Given that there was no detection on subsequent 10 nights, the duty cycle reduces to $5.8 \%$.

We see a dramatic flare in the LRIS data 5 days (UT 2007 June 15) after the high-energy emission, even though the peak magnitude is much fainter. The brightest observed flare in $R$-band was 2 mag in three minutes (see Fig. 8). Much like the behavior seen in X-rays $(\S 2)$, such dramatic optical variability at late times is unlike anything seen before from an extragalactic GRB optical afterglow. Unfortunately none of our optical data directly overlap the XRT light curve, making a direct correlation between the two impossible.

TABLE 2

Optical Observations of Swift J195509.6+261406 with Palomar 60 Inch Telescope

\begin{tabular}{cccccc}
\hline \hline $\begin{array}{c}\text { Epoch } \\
(2007 \text { UT })\end{array}$ & Facility & Filter & $\begin{array}{c}\text { Time Since Burst } \\
(\mathrm{hr})\end{array}$ & $\begin{array}{c}\text { Exposure } \\
(\mathrm{s})\end{array}$ & Magnitude \\
\hline Jun $12.2416 \ldots \ldots \ldots$. & P60 & $i^{\prime}$ & 32.92 & $30.0 \times 1$ & $19.1 \pm 0.14$ \\
Jun $12.2422 \ldots \ldots \ldots .$. & P60 & $i^{\prime}$ & 32.94 & $30.0 \times 1$ & $>19.8$ \\
Jun $12.2433 \ldots \ldots \ldots$. & P60 & $i^{\prime}$ & 32.96 & $30.0 \times 3$ & $>19.8$ \\
Jun $12.2444 \ldots \ldots \ldots .$. & P60 & $i^{\prime}$ & 32.99 & $30.0 \times 5$ & $19.7 \pm 0.16$ \\
Jun $12.2450 \ldots \ldots \ldots$. & P60 & $i^{\prime}$ & 33.00 & $30.0 \times 5$ & $19.6 \pm 0.15$
\end{tabular}

Notes.-Zero point computed in the AB system. The UT epoch denotes the start of observations. Errors quoted are $1 \sigma$ photometric, and instrumental errors summed in quadrature. Note that the uncertainty in the zero-point estimate (relative to USNO-B stars) dominates but is an overestimate for variability studies using relative magnitude. Upper limits quoted are $3 \sigma$. No correction has been made for the large line-of-sight extinction. Table 2 is published in its entirety in the electronic edition of the Astrophysical Journal. A portion is shown here for guidance regarding its form and content. 
TABLE 3

Optical Observations of Swift J195509.6+261406 at Keck I and Palomar Hale

\begin{tabular}{ccccc}
\hline \hline $\begin{array}{c}\text { Mean Epoch } \\
(2007 \text { UT) }\end{array}$ & Facility & Filter & $\begin{array}{c}\text { Exposure } \\
(\mathrm{s})\end{array}$ & $\begin{array}{c}\text { Magnitude } \\
(\mathrm{s})\end{array}$ \\
\hline Jun $13.570 \ldots \ldots \ldots \ldots . \ldots$ & LRIS & $I$ & $120 \times 3$ & $>24.0$ \\
Jun $15.517 \ldots \ldots \ldots \ldots . .$. & LRIS & $I$ & $200 \times 3$ & $24.37 \pm 0.21$ \\
Jun $15.524 \ldots \ldots \ldots \ldots .$. & LRIS & $R$ & $180 \times 1$ & $22.25 \pm 0.06$ \\
Jun $15.527 \ldots \ldots \ldots \ldots . .$. & LRIS & $R$ & $180 \times 1$ & $24.21 \pm 0.13$ \\
Jun $15.531 \ldots \ldots \ldots \ldots . .$. & LRIS & $R$ & $180 \times 1$ & $23.28 \pm 0.07$ \\
Jun $15.534 \ldots \ldots \ldots \ldots .$. & LRIS & $R$ & $180 \times 1$ & $24.09 \pm 0.11$ \\
Jun $15.594 \ldots \ldots \ldots \ldots$. & LRIS & $R$ & $45 \times 8$ & $>25.0$ \\
Aug $13.336 \ldots \ldots \ldots \ldots$. & LRIS & $R$ & $300 \times 4$ & $>26.0$ \\
Sep $13.362 \ldots \ldots \ldots \ldots .$. & LFC & $i^{\prime}$ & $360 \times 26$ & $>24.5$ \\
\hline
\end{tabular}

Note-Zero points computed in the Vega system. Errors quoted are $1 \sigma$ photometric, and instrumental errors summed in quadrature. Upper limits quote are $3 \sigma$. No correction has been made for the large line-of-sight extinction.

Two months after the burst, the optical counterpart faded in $R$-band to fainter than 26.0 and 3 months after the burst, faded in $i^{\prime}$-band to fainter than 24.5 (see Table 3).

\section{A NEAR-INFRARED COUNTERPART}

Given the large line-of-sight extinction, we undertook late-time NIR imaging at a variety of facilities to search for a quiescent counterpart to Swift J195509.6+261406. The results of our campaign are summarized in Table 4.

In detail, we observed the field of Swift J195509.6+261406 with the Near InfraRed Imager and spectrograph (NIRI; Hodapp et al. 2003) mounted on the $8 \mathrm{~m}$ Gemini North Telescope on two occasions. On 2007 June 19 we obtained $18 \times 60$ s images in the $K$-band under exquisite seeing $\left(\sim 0.4^{\prime \prime}\right)$ and photometric conditions. The observations on UT 2007 July 15 suffered from poor seeing and clouds.

On UT 2007 June 21, starting $13: 10$, we observed the transient with Laser Guide Star Adaptive Optics (LGS-AO; Wizinowich et al. 2006; van Dam et al. 2006) on the Keck II Telescope and the Near-Infrared Camera 2 (NIRC2). A total of 17 images were obtained, each consisting of three $20 \mathrm{~s}$ co-added integrations, in the $K^{\prime}$ filter using the wide-angle camera. We also obtained further late-time observations on UT 2007 September 21 and 30.

Finally, $J$ - and $H$-band images were obtained with the WideField Infrared Camera (WIRC; Wilson et al. 2003) mounted on the Palomar Hale 200-inch (P200) telescope. Thirty-four images each with integration time of $30 \mathrm{~s}$ were taken in each filter on the night of UT 21 June 2007.

All but the LGS data were processed with standard IRAF routines. Custom routines in Python and IDL (written by J. S. B. and L.P.) were used for the LGS-AO reductions; a custom distortion correction (obtained by P. B. C. ${ }^{21}$ ) was applied to the LGS-AO imaging. We created an astrometric solution using our Gemini/ NIRI $K$-band image from the night of June 19 relative to about 50 point sources from the Two Micron All-Sky Survey (2MASS; Skrutskie et al. 2006). The resulting rms positional uncertainty was $0.125^{\prime \prime}$ in right ascension and $0.098^{\prime \prime}$ in declination. This NIRI $K$-band image was then used to create a catalog of about 100 point sources for astrometric matching with all other images. The NIRI $K$-band image was chosen because of the excellent seeing conditions $\left(\sim 0.4^{\prime \prime}\right)$ and the larger field of view in comparison to NIRC2. Typical rms positional uncertainties relative to the reference image were $\approx 0.07^{\prime \prime}$ in each coordinate. Using these astrometric solutions,

\footnotetext{
${ }^{21}$ See http://www2.keck.hawaii.edu/inst/n2TopLev/post_observing/dewarp.
}

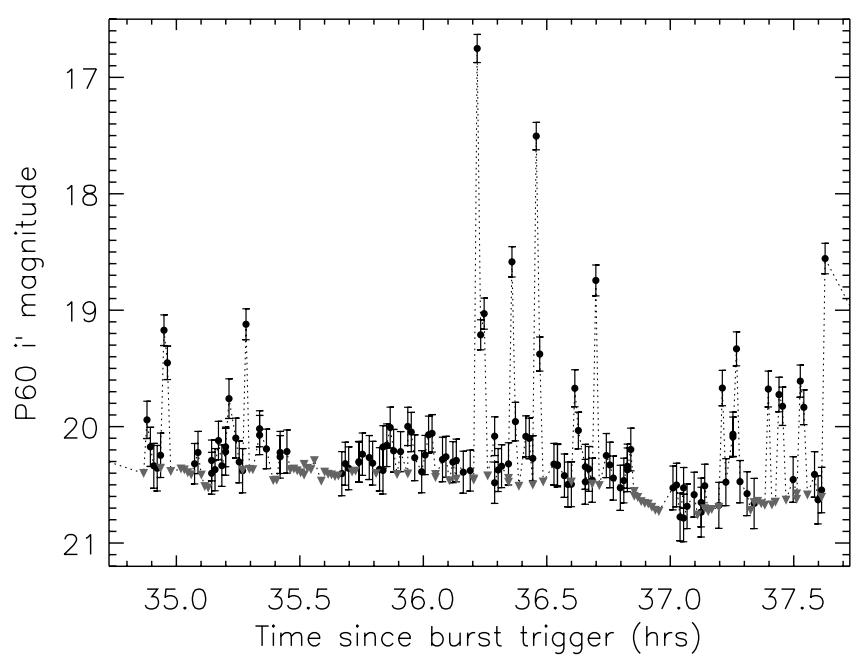

FIG. 7.-P60 $i^{\prime}$-band light curve from the night of 2007 June 12. Upper limits are indicated by gray inverted triangles. The rapid variability (timescales less than $60 \mathrm{~s}$, our sampling rate) at late times is unlike any previous long-duration GRB optical afterglow.

we determine a position for the optical transient in the Keck $R$-band flares of $\alpha=19^{\mathrm{h}} 55^{\mathrm{m}} 09.646^{\mathrm{s}}, \delta=+26^{\circ} 14^{\prime} 05.62^{\prime \prime}$ (J2000.0).

Despite the presence of two nearby objects (A and B), our astrometric accuracy is sufficient to unambiguously identify the $K$-band counterpart to Swift J195509.6+261406 ( $X$ in Fig. 9). Using the LGS-AO/NIRC2 image, we find that the location of this NIR counterpart is $\alpha=19^{\mathrm{h}} 55^{\mathrm{m}} 9.649^{\prime \prime}, \delta=+26^{\circ} 14^{\prime} 5.65^{\prime \prime}$ (J2000.0), with an uncertainty of 100 mas in each direction.

Due to the crowded field, PSF-matched photometry was performed on all images using the IRAF DAOPHOT package. We summarize our NIR observations in Table 4.

For reference, the $R I J H K_{s}$ magnitudes of two extremely nearby objects, A and B, are provided in Table 5. Our late-time data, over 3.5 months after the burst, constrains the quiescent counterpart to be fainter than $K^{\prime}>21.5$ (see Fig. 9).

\section{SEARCH FOR A RADIO COUNTERPART}

On 2007 June 15 we undertook Very Large Array (VLA) 22 observations of Swift J195509.6+261406. The observations were obtained in $2 \times 50 \mathrm{MHz}$ bands around $8.46 \mathrm{GHz}$ and lasted about an hour.

We observed 1956+283 (a phase calibrator) for 0.8 minutes and then switched to Swift J195509.6+261406 for 4.8 minutes. The sequence ended with a 6 minute observation of $0137+331$ (3C 48; flux calibrator).

Data were analyzed using the Astronomical Image Processing System (AIPS) software of National Radio Astronomy Observatory (NRAO). VLA antennas N16, W64, E72, and W48 and baseline combinations EVLA antennas E16, W24, N64, W40, E56, $\mathrm{W} 48$, and N40 were flagged. In total, flagging resulted in a loss of about 100 baselines.

Owing to the VLA being in the " $A$ " configuration, we obtained excellent image resolution of $0.42^{\prime \prime} \times 0.21^{\prime \prime}$. However, Swift J195509.6+261406 was not detected and we get an upper limit of $7.3 \pm 31.5 \mu \mathrm{Jy}$.

\section{ARCHIVAL OBSERVATIONS}

A query of the Simbad database reveals no cataloged object within the BAT localization. The INTEGRAL observatory conducts

22 The National Radio Astronomy Observatory is a facility of the National Science Foundation operated under cooperative agreement by Associated Universities, Inc. 


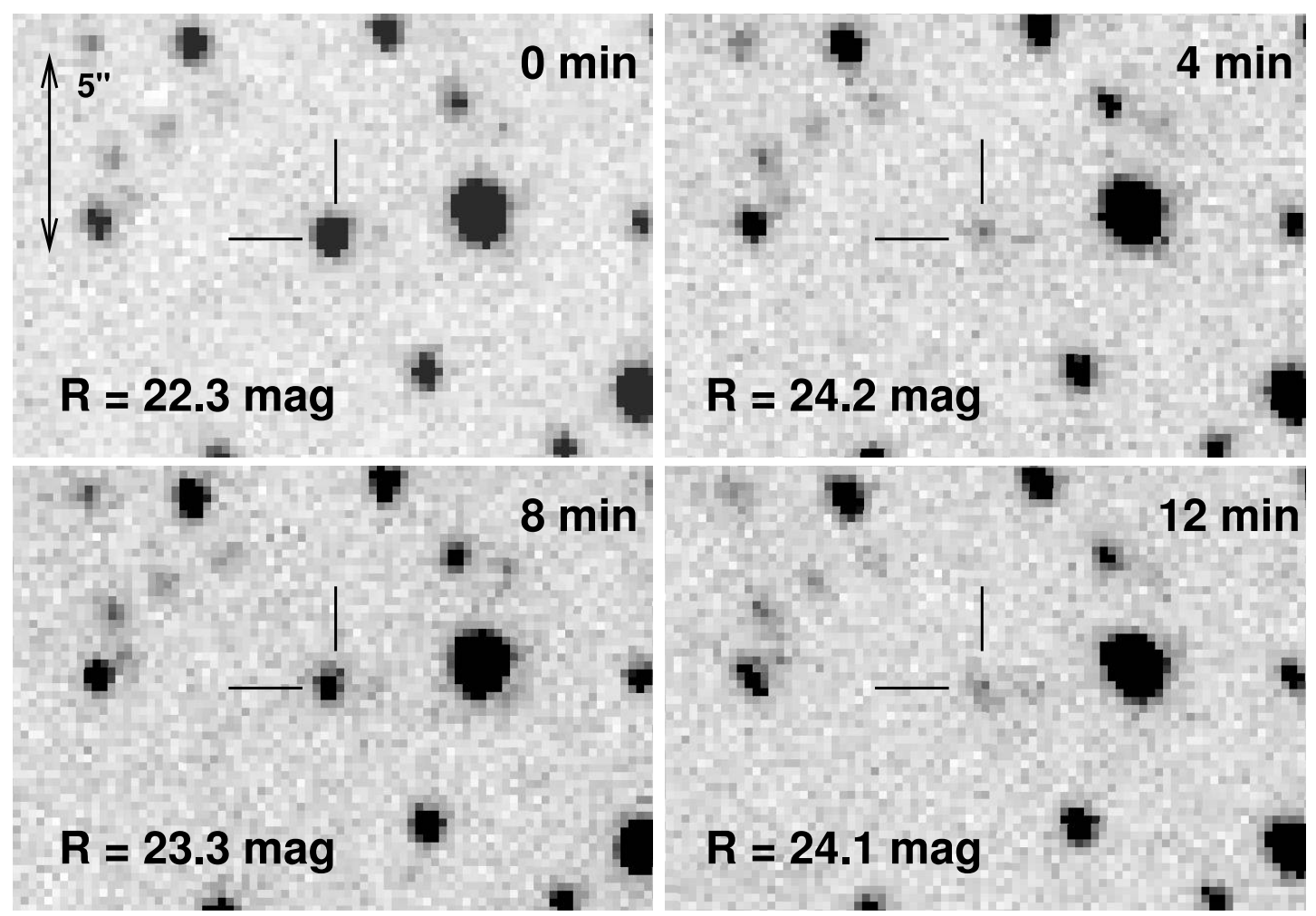

FIG. 8. - Close-up view of the optical field of the Swift J195509.6+261406 optical transient using the LRIS instrument on the Keck I $10 \mathrm{~m}$ telescope; 2007 June 15 starting at $12: 33$ UT. All four images were taken in the $R$-band with a 180 s exposure in sequence. The transient brightens by over 2 mag in only 3 minutes about 5 days after the burst trigger. Such rapid variability at late times is unprecedented from an extragalactic GRB optical afterglow.

regular scans of the Galactic plane and, in addition, performed several long pointed observations of the field around Swift J195509.6+261406. Over the past 4 years, this field has been observed with the IBIS instrument to total $1.5 \mathrm{Ms}$ being within its fully coded field of view $\left(\mathrm{FCFOV}, 9^{\circ} \times 9^{\circ}\right)$ and up to $2.5 \mathrm{Ms}$ being within the partially coded field of view $\left(29^{\circ} \times 29^{\circ}\right)$. The efficiency of observations within the FCFOV falls to zero at the field's edge. The coverage of these 4 years by observations was nonuniform with the maximum exposure reached in the fall of 2006 (for FCFOV).

There is no reported source close to the transient's position in the recent IBIS/ISGRI soft gamma-ray catalogs (Krivonos et al. 2007; Bird et al. 2007). We have also reanalyzed the archival data of INTEGRAL and failed to detect the source. A $4 \sigma$ limit of 0.9 mcrab in the $18-45 \mathrm{keV}$ band (or 0.8 mcrab in the $17-60 \mathrm{keV}$ band) has been received (flux of 1 mcrab corresponds to 1.1 and

TABLE 4

NIR Observations of Swift J195509.6+261406

\begin{tabular}{cccc}
\hline \hline $\begin{array}{c}\text { Epoch } \\
(2007 \text { UT })\end{array}$ & Facility & Filter & Magnitude \\
\hline Jun $19.549 \ldots \ldots \ldots \ldots \ldots$. & Gemini-N/NIRI & $K$ & $19.30 \pm 0.23$ \\
Jun $21.220 \ldots \ldots \ldots \ldots \ldots$. & Keck II/LGS-AO+NIRC2 & $K^{\prime}$ & $19.83 \pm 0.15$ \\
Jul $15.309 \ldots \ldots \ldots \ldots \ldots$. & Gemini-N/NIRI & $K$ & $>19.5$ \\
Jun $21.352 \ldots \ldots \ldots \ldots \ldots$. & P200/WIRC & $J$ & $>20.5$ \\
Jun $21.400 \ldots \ldots \ldots \ldots \ldots$. & P200/WIRC & $H$ & $>19.5$ \\
Sep $21.632 \ldots \ldots \ldots \ldots \ldots$. & Keck II/LGS-AO+NIRC2 & $K^{\prime}$ & $>20.3$ \\
Sep $30.264 \ldots \ldots \ldots \ldots \ldots$. & Keck II/LGS-AO+NIRC2 & $K^{\prime}$ & $>21.5$ \\
\hline
\end{tabular}

NoTES.-Errors quoted are $1 \sigma$ photometric and instrumental errors summed in quadrature. Upper limits quoted are $3 \sigma$. No correction has been made for the large line-of-sight extinction.
$1.4 \times 10^{-11} \mathrm{ergs} \mathrm{cm}^{-2} \mathrm{~s}^{-1}$ in these bands respectively for a source with Crab-like spectrum). There was also no source detected on a timescale of one individual pointing (2.0-3.6 ks). We derive a $4 \sigma$ limit of $\sim 20$ mcrab.

Spitzer observed the position of Swift J195509.6+261406 during the Galactic Legacy Infrared Mid-Plane Survey Extraordinaire (GLIMPSE) on 2004 October 31. Conservative upper limits for a source at the $K$-band position are $280,350,1700$, and $6350 \mu \mathrm{Jy}$ at $3.6,4.5,5.8$, and $8.0 \mu \mathrm{m}$, respectively.

\section{BASIC CONSIDERATIONS: DISTANCE, ENERGETICS AND RADIUS}

The fluence, the hardness and the duration of GRB 070610 are not atypical of GRBs. However, Swift J195509.6+261406 is an atypical afterglow in the X-ray band $(\S 2)$. The optical counterpart is also atypical. Correcting for the total interstellar extinction along the line of sight, the apparent $i^{\prime}$-band magnitude of the optical transient is as bright as $\sim 13$ mag more than a day after the burst trigger - there is no other optical extragalactic afterglow as bright at such late times.

The issue that faces us is quite simple: is GRB 070610 related to Swift J195509.6+261406? For extragalactic long-duration GRBs that the Swift-XRT was able to observe within an hour of the burst trigger, the overwhelming majority have a detected $\mathrm{X}$-ray afterglow.

We therefore consider it unlikely that GRB 070610 arises from a background (i.e., extragalactic) event. The spatial and temporal coincidence of GRB 070610 and Swift J195509.6+ 261406 suggest that these are strongly related. If so, the event is of Galactic origin. Accepting this association we turn our attention to the fundamental parameters characterizing Swift J195509.6+261406. 


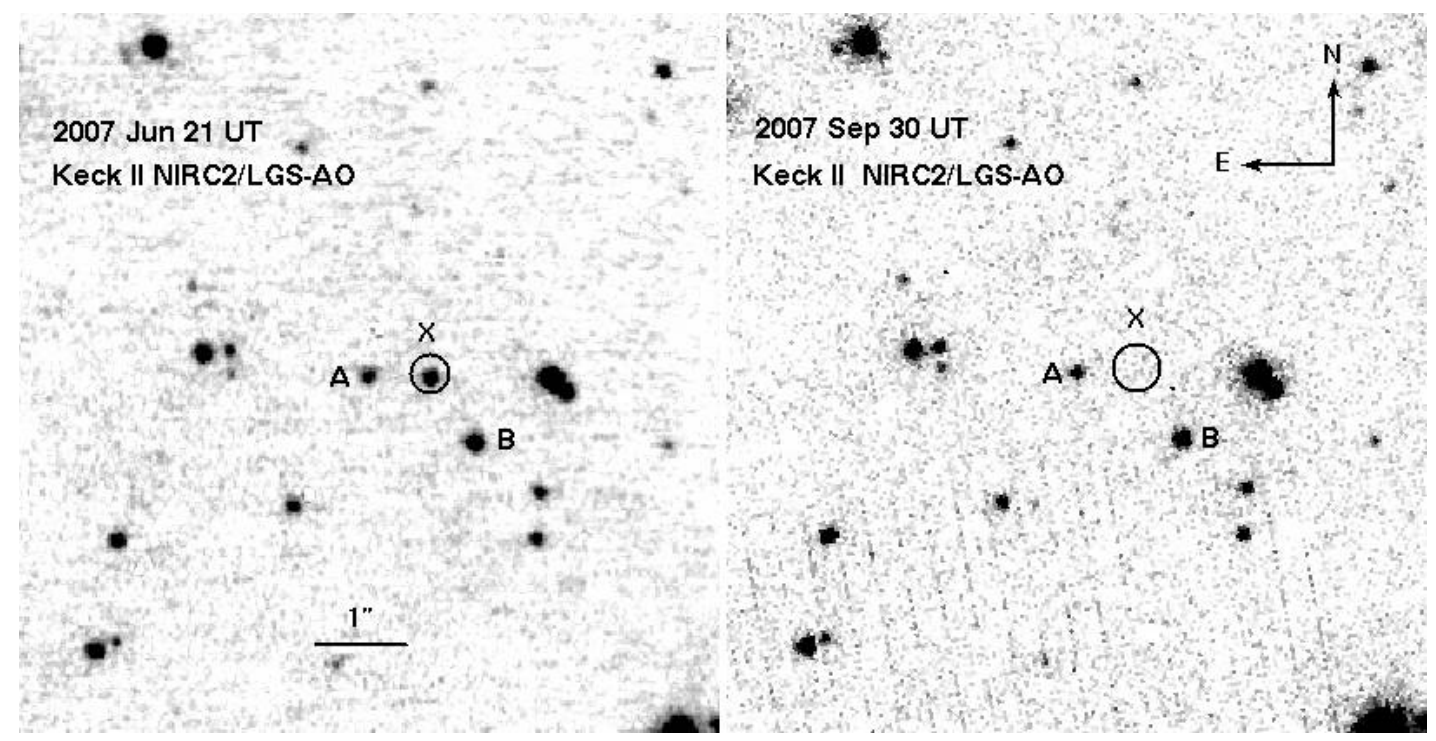

FIG. 9. - K-band image of the field of Swift J195509.6+261406 obtained with NIRC-2 imager behind the Keck II Laser Guide Star (LGS) system on 2007 June 21 (left) and 2007 September 30 (right). The $2 \sigma$ error circle of the optical transient (taken from our LRIS imaging) is shown as a black circle overlaid on the LGS image. Clearly we can identify the object marked by the cross as the NIR counterpart of Swift J195509.6+261406.

The extinction estimate based on the full X-ray spectrum excluding the flare (see Table 1) corresponds to $E(B-V)=1.0-$ $1.5 \mathrm{mag}$ [based on optical spectral classification of nearby stars, we find $E(B-V) \sim 1.1]$. Assuming $R=3.1$, this corresponds to $A_{K}=0.4-0.5 \mathrm{mag}$. The infrared $K^{\prime}$-band magnitude of the NIR counterpart Swift J195509.6+261406 is no brighter than $\sim 21.5$ (Table 4). From late-time optical observations, we also know that $R>26.0$ (Table 3 ). Since the farthest distance for a star in the disk of our galaxy is $30 \mathrm{kpc}$, we get extinction-corrected absolute magnitude of $M_{K}>3.6$ and $M_{R}>4.8$. This clearly rules out the luminosity class of giants and supergiants (Cox 2000). This also constrains the spectral type to be cooler than G8 (Kraus $\&$ Hillenbrand 2007). If we assume a distance of $10 \mathrm{kpc}$, we can further constrain it to a spectral type cooler than M3.

The prompt gamma-ray burst peak flux is $5 \times 10^{-8} \mathrm{ergs} \mathrm{cm}^{-2}$ $\mathrm{s}^{-1}$, the peak X-ray flare flux is twice as faint and the mean flux over the first week is approximately a factor of $10^{4}$ fainter than the burst peak flux. These translate to the following isotropic luminosities: $6 \times$ $10^{38} d_{10}^{2} \mathrm{ergs} \mathrm{s}^{-1}, 3 \times 10^{38} d_{10}^{2} \mathrm{ergs} \mathrm{s}^{-1}$ and $5 \times 10^{34} d_{10}^{2} \mathrm{ergs} \mathrm{s}^{-1}$.

The prompt gamma rays can constrain the radius of the emission. The BAT burst duration of several seconds (see Fig. 1) puts an upper limit on the size of the emitting region (along the line of sight) to be smaller than $\sim 10^{11} \beta_{\mathrm{c}} \mathrm{cm}$, where $\beta_{c}$ is the causal speed in units of the light speed (i.e., the speed in which informa-

TABLE 5

Photometry of Nearby Contaminating Sources $A$ and $B$

\begin{tabular}{|c|c|c|c|c|}
\hline \multirow{2}{*}{$\begin{array}{c}\text { Еросн } \\
(2007 \text { UT) }\end{array}$} & \multirow[b]{2}{*}{ FACILITY } & \multirow[b]{2}{*}{ FILTER } & \multicolumn{2}{|c|}{ MAGNITUdE } \\
\hline & & & Source A & Source B \\
\hline Jun $15.594 \ldots \ldots \ldots \ldots \ldots$ & Keck I/LRIS & $R$ & $>25.0$ & $>25.0$ \\
\hline Aug $13.336 \ldots \ldots \ldots \ldots . . .$. & Keck I/LRIS & $R$ & $>26.0$ & $>26.0$ \\
\hline Jun $15.517 \ldots \ldots \ldots \ldots \ldots . . . .$. & Keck I/LRIS & $I$ & $24.83 \pm 0.21$ & $24.93 \pm 0.22$ \\
\hline Jun $21.352 \ldots \ldots \ldots \ldots \ldots . . .$. & P200/WIRC & $J$ & $>20.5$ & $>20.5$ \\
\hline Jun $21.400 \ldots \ldots \ldots \ldots . . .$. & P200/WIRC & $H$ & $>19.5$ & $>19.5$ \\
\hline Jun $21.220 \ldots \ldots \ldots \ldots \ldots$ & Keck II/LGS & $K^{\prime}$ & $20.30 \pm 0.16$ & $19.44 \pm 0.14$ \\
\hline
\end{tabular}

Notes.-Source B is $471 \pm 22$ mas West wand $670 \pm 22$ mas south of Swift $\mathrm{J} 195509.6+261406$. In images with poorer angular resolution, stars A and B may contaminate the photometry of the transient (i.e., our NIRI imaging). tion, such as sound, travels). Since we expect $\beta_{c} \ll 1$ in noncompact objects (e.g., main sequence stars) the source of the prompt gamma rays is a black hole, a neutron star or a white dwarf (the sound crossing time of the latter is seconds).

On the other hand, the nonthermal gamma rays can be used to put a lower limit on the emission radius. If the gamma-ray spectrum continues to high energy $\left(E>m_{e} c^{2}\right)$ then pair production opacity starts playing a role. Using the formulation of Lithwick \& Sari (2001) and assuming a nonrelativistic source we find that the size of the emitting region has to be $\gtrsim L \sigma_{T} /\left(0.1 \pi m_{e} c^{3}\right) \approx$ $10^{9} d_{10}^{2} \mathrm{~cm}$, where the approximated numerical factor (taken here as $0.1 \pi$ ) depends on the radiation spectrum and the geometry of the source (Svensson 1987). This radius implies that if the engine of the burst is a neutron star or a black hole then the observed gamma rays are produced far from the engine by (possibly relativistic) ejecta.

\section{A CURIOUS GALACTIC TRANSIENT}

With a compact object ( $\S 7)$ and a fainter than K-dwarf companion, Swift J195509.6+261406 is likely a binary system. We now turn our attention to investigate the mechanism powering the unusual emission, with an emphasis on identifying analogous systems in our Galaxy.

At first blush, soft gamma-ray repeater (SGR) flares appear to be a viable model for Swift J195509.6+261406. SGR exhibits hard $\mathrm{X}$-ray flares with durations ranging from 0.1 to $10 \mathrm{~s}$ and isotropic luminosities of $10^{46}$ ergs (Aptekar et al. 2001; Hurley et al. 2005). Furthermore, variable X-ray afterglows have been detected after several SGR outbursts (see, e.g., Woods \& Thompson 2006).

However, this interpretation has several problems. First, SGR flares lasting longer than $1 \mathrm{~s}$, dubbed "intermediate" SGR flares, have an energy release $\approx 10^{41}$ ergs, 2 orders of magnitude larger than our upper limit for Swift J195509.6+261406 (Woods \& Thompson 2006). Second, pulsations are typically observed in SGR flare X-ray afterglows at the neutron star spin rate. We see no evidence for pulsations from Swift J195509.6+261406 (although constrained by the $2.5 \mathrm{~s}$ sampling interval). Finally, no known SGR has a companion. If Swift J195509.6+261406 were caused by an SGR flare, a cooler than K-dwarf companion would make Swift J195509.6+261406 the first binary magnetar. 
Unlike SGR flares, the remaining possibilities are ultimately powered by accretion instead of magnetic activity (Arefiev et al. 2003 provide a comprehensive review of such transients in the hard X-ray sky). Cygnus X-1, a black hole binary with a supergiant companion, exhibits hard X-ray outbursts (Stern et al. 2001; Golenetskii et al. 2003). The INTEGRAL mission has identified a class of bright hard X-ray transients, the so-called supergiant fast X-ray transients (SFXTs; Negueruela et al. 2007). However, these events are relatively soft, and have timescales of $10^{3} \mathrm{~s}$ or longer. Furthermore, the supergiant donor is an essential part of the SFXT story - the X-ray flares arise from accretion of "blobs" in the wind of the supergiant star. The faintness of the quiescent counterpart convincingly rules out the giant and supergiant scenarios.

The high peak luminosity strongly suggests an event like CI Cam (see Belloni et al. 1999). However, this too is a questionable analog for the reasons of the lack of a bright optical/NIR counterpart and also the short flare duration. For the same reasons, the analogy to A0538-66 (the well-known Be-pulsar X-ray binary in the LMC) can also be ruled out.

The bursting pulsar GRO J1744-28 shares some properties with those from GRB 070610. From 1995 to 1997, thousands of bursts were detected by BATSE out to $>60 \mathrm{keV}$ (Kouveliotou et al. 1996; Woods et al. 1999). The spectrum of the bursts in BATSE and $R X T E$ was adequately modeled by a thermal bremsstrahlung model having $k T \sim 10 \mathrm{keV}$; burst durations were approximately 10 s. GRO J1744-28 consists of a neutron star in an 11.8 day orbit with a low-mass companion (Finger et al. 1996). However, unlike Swift J195509.6+261406, there is no evidence for a highly variable optical emission associated with these bursts. Also the high-energy bursts from GRO J1744-28 are highly repetitive. Searches for other episodes of emission from GRB $070610 \mathrm{did}$ not yield any obvious candidates either in the BAT data or in the extensive INTEGRAL survey ( $(6)$ and the Interplanetary Network (§ 9).

The best analog to the X-ray and optical emission from Swift J195509.6+261406 is V4641 Sgr (Markwardt et al. 2007) - a transient which has been recognized by several authors as being one of the fastest transients in the hard X-ray (in 't Zand et al. 2000; Uemura et al. 2002; Arefiev et al. 2003). V4641 Sgr came to the attention of astronomers through a major outburst in 1999 (see in 't Zand et al. 2000). We now infer that this object is a binary consisting of a B9 III star orbiting a $9 M_{\odot}$ black hole (Orosz et al. 2001). The system exhibited strong and fast X-ray and optical variability - similar to what we see in Swift J195509.6+261406.

Rapid $(<100$ s) and intense (modulation index, $S=\langle f\rangle /$ $\Delta(f) \gtrsim 10$; here $f$ is the X-ray flux and $\Delta f$ is the variability in $f$ ) but with mean X-ray luminosity $\langle L\rangle$ that is well below Eddington flux mark V4641 Sgr from the other black hole binaries (Revnivtsev et al. 2002). The classical black hole LMXBs such as A0620-00 exhibit X-ray novae with peak super-Eddington flux and a decline over a month (see reviews by Tanaka \& Shibazaki 1996; Remillard \& McClintock 2006). Microquasars such as GRS 1915+10 exhibit intense variations (with $S$ approaching 10) but only when $\langle L\rangle$ is extremely high, $\langle L\rangle \sim 10^{39} \mathrm{ergs} \mathrm{s}^{-1}$ (e.g., Belloni et al. 1997; Muno et al. 1999).

The first difference between Swift J195509.6+261406 and V4641 Sgr is the donor star: Swift J195509.6+261406 has a cool dwarf donor, while V4641 Sgr has a B9 giant donor. We suggest that the distinctive variability of V4641 Sgr arises from the black hole companion and has less to do with the nature of the donor star. This conjecture would allow us to infer that the compact object in Swift J195509.6+261406 is also a black hole. While V4641 Sgr seems to be the closest event we have to Swift J195509.6+ 261406 , it is clear that no perfect analog to Swift J195509.6+
261406 exists. In particular, there has been no report of a burst of gamma rays from V4641 Sgr. However, the absence could be due to the short duration duty cycle of the gamma-ray bursts.

With two similar objects in hand-V4641 Sgr and GRB 070610 - we now have the luxury of defining a new class of transients: fast X-ray novae which, in addition to the rapid X-ray and optical variability but at sub-Eddington luminosities, are also (apparently) marked by GRB-like bursts.

What differentiates fast X-ray novae from the regular X-ray novae? Regular X-ray novae are essentially black hole binaries undergoing the equivalent of dwarf novae i.e., instabilities in the disk. During the major burst of 1999, V4641 Sgr exhibited radio emission and relativistic motion (Hjellming et al. 2000). The radio flux of V4641 Sgr declined very steeply initially; from $360 \mathrm{mJy}$ at $8.3 \mathrm{GHz}$ to $30 \mathrm{mJy}$ at $8.46 \mathrm{GHz}$ in one day (Hjellming et al. 2000). According to Orosz et al. 2001, the distance to V4641 Sgr is 7.4$12.3 \mathrm{kpc}$ and the apparent expansion velocity is $>9.5 \mathrm{c}$ (assuming the lowest proper motion estimate from Hjellming et al. 2000)making V4641 Sgr the most relativistic of Galactic sources. This suggests that perhaps the key difference between fast X-ray novae and the regular X-ray novae is the speed at which the ejecta is emitted. Unfortunately, neither was GRB 070610 as bright as V4641 Sgr in the optical and X-ray immediately after the flare nor were our radio observations undertaken promptly after the detection of GRB 070610 to verify this hypothesis.

\section{IMPLICATIONS: GALACTIC GRBs}

Spurred by the connection between Swift J195509.6+261406 with a Galactic transient we investigated whether this source or its analog V4641 Sgr emitted bursts of gamma rays in the past. We have constructed a list of 1211 GRBs detected by the IPN (Hurley et al. 1999), whose position is constrained by at least one annulus with semiwidth smaller than $0.5^{\circ}$. This catalog contains events observed from 1990 November 12 to 2005 October 31 (see Ofek 2007 for more details). We did not find any IPN GRB that coincides with either of these positions.

We also searched for Swift-BAT subthreshold events which are consistent with the positions of V4641 Sgr and Swift J195509.6+ 261406. There is no BAT subthreshold event within $5^{\prime}$ from the location of V4641 Sgr. But, we find an event at a signal-to-noise ratio $(\mathrm{S} / \mathrm{N})$ of 5.0 located at R.A. $=298.77^{\circ}$, decl. $=+26.221^{\circ}$ (1.8 from the position of GRB 070610) and occurring on UT 2006 Nov 17.7812. However, adjusted for the approximate number of times this field has been observed, the significance drops below $2 \sigma$. We consider it likely this subthreshold event is nothing more than a statistical fluctuation.

V4641 Sgr has been undergoing major bursts approximately every 2 years (see Uemura et al. 2004). The absence of a detection of a gamma-ray burst could simply be due to lack of coverage or that not all such bursting activity are preceded by a burst of gamma rays.

Another possible member of this class of fast X-ray novae is XTE J1901+014 (Karasev et al. 2007), which is potentially associated with GRB 020406 (Remillard \& Smith 2002).

Nonetheless, it is reasonable to speculate that a burst similar to Swift J195509.6+261406 occurs in our Galaxy, say, every decade. This alone immediately makes Swift J195509.6+261406 and related events as the most common of long-duration gamma-ray bursts. (The mean time between cosmological GRBs in our Galaxy is no smaller than $10^{5} \mathrm{yr}$ ).

Several hundred years ago, optical astronomers called all new apparitions of stars novae stella. Over the past century astronomers have shown that novae stella split into three distinctly different phenomena: novae, supernovae of Type Ia, and core-collapse 
supernovae. The novae, in turn, are divided into five families which arise from instabilities in the accretion disk feeding a white dwarf, neutron star or a black hole and on the surfaces of white dwarfs and neutron stars.

History is repeating itself. Only 30 years ago, astronomers referred to all bursts of gamma-ray radiation as GRBs. Over the last decade astronomers have established SHBs and LSBs to be of cosmological origin (Metzger et al. 1997; Gehrels et al. 2005; Bloom et al. 2006; Fox et al. 2005) and reasonably established their origin: coalescence of compact objects and deaths of massive stars, respectively.

However, fissures are already developing. Recently, hypergiant flares from magnetars in our own Galaxy and nearby galaxies have been found to contaminate the SHB sample. The Galactic rate of the hypergiant flares is likely $10^{-3} \mathrm{yr}^{-1}$ (Ofek 2007), much larger than the estimated Galactic SHB rate of $10^{-6} \mathrm{yr}^{-1}$ (Nakar et al. 2006; Guetta \& Piran 2006).

Our galaxy has at least two fast X-ray novae systems (V4641 Sgr and Swift J195509.6+261406). The rate of GRB 070610like events (with no assumption about beaming) is likely to be about $3.5_{-2.9}^{+8.0} \mathrm{yr}^{-1}$ which is 5 orders of magnitude larger than the estimated cosmological GRBs rate. However, these events are yet unobservable outside our galaxy with the current limitation in sensitivity of high-energy detectors. As usual the meekest events dominate the demography.

We thank M. van Kerkwijk for help with Keck observations and discussions. We thank J. Cohen, J. Simon, A. Kraus, M. Muno, E. S. Phinney, and R. Narayan for valuable discussions. We also acknowledge D. Law, T. Treu, and P. Marshall. As always, we are grateful to the selfless librarians and astronomers who maintain the Simbad database. M. M. K. thanks the Gordon and Betty Moore Foundation for support with the George Ellory Hale Fellowship. S. B. C. and A. M. S. are supported by a NASA Graduate Student Research Fellowship. J. S. B. is a Sloan Research Fellow and is partially supported by a Hellman Faculty Award. P. C. S. is supported by a Jansky Fellowship. E. B. is supported by a Hubble fellowship. GRB research at Caltech is supported in part by grants from NSF (AST program) and NASA (Swift and HST missions).

Facilities: PO:1.5m, Hale, Keck:I, Keck:II, VLA, Swift
Aptekar, R. L., Frederiks, D. D., Golenetskii, S. V., Il'inskii, V. N., Mazets, E. P., Pal'shin, V. D., Butterworth, P. S., \& Cline, T. L. 2001, ApJS, 137, 227

Arefiev, V. A., Priedhorsky, W. C., \& Borozdin, K. N. 2003, ApJ, 586, 1238

Barthelmy, S. D., et al. 2005, Space Sci. Rev., 120, 143

Belloni, T., Mendez, M., King, A. R., van der Klis, M., \& van Paradijs, J. 1997, ApJ, 488, L109

Belloni, T., et al. 1999, ApJ, 527, 345

Bird, A. J., et al. 2007, ApJS, 170, 175

Bloom, J. S., et al. 2006, ApJ, 638, 354

Buccheri, R., et al. 1983, A\&A, 128, 245

Burrows, D. N., et al. 2005, Space Sci. Rev., 120, 165

Cenko, S. B., et al. 2006, PASP, 118, 1396

Chincarini, G., et al. 2007, ApJ, 671, 1903

Cox, A. N. 2000, Allen's Astrophysical Quantities, ed. A. N. Cox (4th ed., New York: Springer)

Dickey, J. M., \& Lockman, F. J. 1990, ARA\&A, 28, 215

Evans, P. A., et al. 2007, A\&A, 469, 379

Falcone, A. D., et al. 2006, ApJ, 641, 1010

Finger, M. H., Koh, D. T., Nelson, R. W., Prince, T. A., Vaughan, B. A., \& Wilson, R. B. 1996, Nature, 381, 291

Fox, D. B., et al. 2005, Nature, 437, 845

French, J., Melady, G., Kubanek, P., \& Jelinek, M. 2007, GCN Circ., 6500, $\mathrm{http} / / / \mathrm{gcn} . \mathrm{gsfc}$.nasa.gov/gen/gcn3/6500.gen3

Gehrels, N., et al. 2004, ApJ, 611, 1005 . 2005, Nature, 437, 851

Golenetskii, S., Aptekar, R., Frederiks, D., Mazets, E., Palshin, V., Hurley, K.,

Cline, T., \& Stern, B. 2003, ApJ, 596, 1113

Guetta, D., \& Piran, T. 2006, A\&A, 453, 823

Hjellming, R. M., et al. 2000, ApJ, 544, 977

Hodapp, K. W., et al. 2003, PASP, 115, 1388

Hurley, K., Briggs, M. S., Kippen, R. M., Kouveliotou, C., Meegan, C., Fishman, G., Cline, T., \& Boer, M. 1999, ApJS, 120, 399

Hurley, K., et al. 2005, Nature, 434, 1098

in 't Zand, J. J. M., et al. 2000, A\&A, 357, 520

Kalberla, P. M. W., Burton, W. B., Hartmann, D., Arnal, E. M., Bajaja, E., Morras, R., \& Pöppel, W. G. L. 2005, A\&A, 440, 775

Kann, D. A., Wilson, A. C., Schulze, S., Klose, S., Henze, M., Ludwig, F., Laux, U., \& Greiner, J. 2007, GCN Circ., 6505, http:/gcn.gsfc.nasa.gov/gcn/ $\operatorname{gcn} 3 / 6505 . g \mathrm{cn} 3$

Karasev, D. I., Lutovinov, A. A., \& Grebenev, S. A. 2007, Astron. Lett., 33, 159

Klotz, K. A., Boer, B. M., Atteia, A. J. L., \& Gendre, G. B. 2007, GCN Circ., 6513, http://gcn.gsfc.nasa.gov/gen/gcn3/6513.gcn3

Kouveliotou, C., van Paradijs, J., Fishman, G. J., Briggs, M. S., Kommers, J.,

Harmon, B. A., Meegan, C. A., \& Lewin, W. H. G. 1996, Nature, 379, 799

Kraus, A. L., \& Hillenbrand, L. A. 2007, AJ, 134, 2340

Krivonos, R., Revnivtsev, M., Lutovinov, A., Sazonov, S., Churazov, E., \& Sunyaev, R. 2007, A\&A, 475, 775

Lithwick, Y., \& Sari, R. 2001, ApJ, 555, 540

Markwardt, C. B., Pagani, C., Evans, P., Gavriil, F. P., Kennea, J. A., Krimm, H. A., Landsman, W., \& Marshall, F. E. 2007, Astron. Tel, 1102, 1
Metzger, M. R., Djorgovski, S. G., Kulkarni, S. R., Steidel, C. C., Adelberger, K. L., Frail, D. A., Costa, E., \& Frontera, F. 1997, Nature, 387, 878

Muno, M. P., Morgan, E. H., \& Remillard, R. A. 1999, ApJ, 527, 321

Nakar, E., Gal-Yam, A., \& Fox, D. B. 2006, ApJ, 650, 281

Negueruela, I., Smith, D. M., Torrejon, J. M., \& Reig, P. 2007, preprint (arXiv: 0704.3224N)

Norris, J. P., Nemiroff, R. J., Bonnell, J. T., Scargle, J. D., Kouveliotou, C., Paciesas, W. S., Meegan, C. A., \& Fishman, G. J. 1996, ApJ, 459, 393

Nousek, J. A., et al. 2006, ApJ, 642, 389

Ofek, E. O. 2007, ApJ, 659, 339

Oke, J. B., et al. 1995, PASP, 107, 375

Orosz, J. A., et al. 2001, ApJ, 555, 489

Pagani, C., Racusin, J. L., \& Kennea, J. A. 2007a, GCN Circ., 6506, http:// gen.gsfc.nasa.gov/gen/gcn3/6506.gen3

2007b, GCN Circ., 6489, http://gcn.gsfc.nasa.gov/gcn/gcn3/6489.gcn3

Postigo, A. d. U., Castro-Tirado, A. J., \& Aceituno, F. 2007, GCN Circ., 6501, http://gen.gsfc.nasa.gov/gen/gen3/6501.gcn3

Remillard, R. A., \& McClintock, J. E. 2006, ARA\&A, 44, 49

Remillard, R. A., \& Smith, D. 2002, Astron. Tel, 88, 1

Revnivtsev, M., Gilfanov, M., Churazov, E., \& Sunyaev, R. 2002, A\&A, 391, 1013

Schlegel, D. J., Finkbeiner, D. P., \& Davis, M. 1998, ApJ, 500, 525

Skrutskie, M. F., et al. 2006, AJ, 131, 1163

Stefanescu, A., Slowikowska, A., Kanbach, G., Duscha, S., Schrey, F., Steinle, H., \& Ioannou, Z. 2007a, GCN Circ., 6492, http:/gcn.gsfc.nasa.gov/gen/gcn3/ 6492.gen3

Stefanescu, A., et al. 2007b, GCN Circ., 6508, http:/gcn.gsfc.nasa.gov/gcn/ gcn3/6508.gcn3

. 2007c, GCN Circ., 6532, http://gcn.gsfc.nasa.gov/gcn/gcn3/6532.gcn3

Stern, B. E., Beloborodov, A. M., \& Poutanen, J. 2001, ApJ, 555, 829

Svensson, R. 1987, MNRAS, 227, 403

Tanaka, Y., \& Shibazaki, N. 1996, ARA\&A, 34, 607

Tueller, J., et al. 2007, GCN Circ., 6491, http://gcn.gsfc.nasa.gov/gcn/gen3/6491.gcn3

Uemura, M., Kato, T., Watanabe, T., Stubbings, R., Monard, B., \& Kawai, N. 2002, PASJ, 54, 95

Uemura, M., et al. 2004, PASJ, 56, 823

Updike, A. C., Hartmann, D. H., Henson, G., et al. 2007a, GCN Circ., 6507, http://gen.gsfc.nasa.gov/gen/gen3/6507.gen3

Updike, A. C., et al. 2007b, GCN Circ., 6536, http://gcn.gsfc.nasa.gov/gcn/ gen $3 / 6536 . g c n 3$

van Dam, M. A., et al. 2006, PASP, 118, 310

Wilson, J. C., et al. 2003, Proc. SPIE, 4841, 451

Wizinowich, P. L., et al. 2006, Proc. SPIE, 6272, 7

Woods, P. M., \& Thompson, C. 2006, in Compact Stellar X-Ray Sources, ed. W. Lewin \& M. van der Klis (Cambridge: Cambridge Univ. Press), 547

Woods, P. M., et al. 1999, ApJ, 517, 431

Yoshida, M., et al. 2007, GCN Circ., 6512, http://gcn.gsfc.nasa.gov/gcn/gcn3/ 6512.gcn3

Zhang, B., Fan, Y. Z., Dyks, J., Kobayashi, S., Mészáros, P., Burrows, D. N., Nousek, J. A., \& Gehrels, N. 2006, ApJ, 642, 354 